\title{
Speed accuracy tradeoff? Not so fast: Marginal changes in speed 1 have inconsistent relationships with accuracy in real-world settings a
}

\author{
Benjamin W. Domingue ${ }^{1, \dagger}$, Klint Kanopka ${ }^{1}$, Ben Stenhaug ${ }^{1}$, Michael J. Sulik ${ }^{1}$, Tanesia ${ }_{3}$ \\ Beverly $^{2,3, a}$, Matthieu Brinkhuis ${ }^{4, a}$, Ruhan Circi $^{5, a}$, Jessica Faul ${ }^{6, a}$, Dandan Liao, a , Bruce ${ }_{4}$ \\ McCandliss $^{1, a}$, Jelena Obradović ${ }^{1, a}$, Chris Piech ${ }^{8, a}$, Tenelle Porter ${ }^{9, a}$, Jim Soland ${ }^{10,11, a}$, Jon ${ }_{5}$ \\ Weeks $^{12, a}$, Steve Wise ${ }^{10, a}$, and Jason Yeatman ${ }^{1, a}$ \\ ${ }^{1}$ Stanford Graduate School of Education 7 \\ ${ }^{2}$ University of Connecticut ${ }_{8}$ \\ ${ }^{3}$ Law School Admissions Council 9 \\ ${ }^{4}$ Utrecht University ${ }_{10}$ \\ ${ }^{5}$ American Institutes for Research $\quad{ }_{11}$ \\ ${ }^{6}$ University of Michigan \\ ${ }^{7}$ Cambium Learning Group $\quad{ }_{13}$ \\ ${ }^{8}$ Department of Computer Science, Stanford University ${ }^{14}$ \\ ${ }^{9} \mathrm{UC}$ Davis $\quad{ }_{15}^{15}$ \\ ${ }^{10}$ NWEA \\ ${ }^{11}$ University of Virginia $\quad{ }_{17}^{17}$ \\ ${ }^{12}$ Educational Testing Service $\quad{ }_{18}$ \\ †bdomingu@stanford.edu $\quad{ }_{19}$ \\ ${ }^{a}$ Alphabetical 20
}

\begin{abstract}
The speed-accuracy tradeoff suggests that responses generated under time constraints will be less accurate. While it has undergone extensive experimental verification, it is less clear whether it applies in settings where time pressures are not being experimentally manipulated (but where respondents still vary in their utilization of time). Using a large corpus of 29 response time datasets containing data from cognitive tasks without experimental manipulation of time pressure, we probe whether the speedaccuracy tradeoff holds within-person across a variety of tasks using idiosyncratic variation in speed. We find inconsistent relationships between marginal increases in time spent responding and accuracy; in many cases, marginal increases in time do not predict increases in accuracy. However, we do observe time pressures (in the form of time limits) to consistently reduce accuracy and for rapid responses to typically show the anticipated relationship (i.e., they are more accurate if they are slower). We also consider analysis of items and individuals. We find substantial variation in the item-level associations between speed and accuracy. On the person side, respondents who exhibit more within-person variation in response speed are typically of lower ability. Finally, we consider the predictive power of a person's response time in predicting out-of-sample responses; it is generally a weak predictor. Collectively, our findings suggest the speed-accuracy tradeoff may be limited as a conceptual model in its application in non-experimental settings and, more generally, offer empirical results and an analytic approach that will be useful as more response time data is collected.
\end{abstract}

Keywords: Response Time, IRT, Speed-accuracy tradeoff 


\section{Introduction}

The speed accuracy tradeoff predicts that time pressure should lead to less accurate responses. When respondents have more time to generate item responses, they should respond more accurately (see the conceptual model in Figure 1). The basic notion of the speed-accuracy tradeoff (SAT) is an intuitively appealing one: more deliberate responses should be more accurate ones. It's appeal lies in the observation that, for an individual, decisions made in the context of ample time should, all else equal, be more accurate than rushed ones. Beyond its intuitive appeal, it has also seen extensive verification work in laboratory settings wherein a variety of manipulations are used to induce changes in speed and has also been investigated in non-human animal models [1]. Further, connections are being made to functioning of the nervous system $[2,3,4]$.

While there is great power in using experimental manipulation of time pressure for identification of this phenomena, experimental results do not necessarily generalize to non-experimental settings where additional factors may impact choice of speed and the resulting level of accuracy. The ubiquity of digital interfaces for all manner of widely varying psychological instruments have rapidly increased the availability of response time data in psychometric settings. This increase in response time data across a variety of psychological measures in observational settings increases the need for models - both conceptual and statistical - for understanding such data and also increases the importance of questions about the generalizability of insights derived from experimental settings.

In settings wherein time pressures are not explicitly being manipulated, the SAT may still be a relevant model of behavior. Earlier work has described this kind of SAT, based on idiosyncratic within-person changes in speed during the measurement process, as a "micro" SAT [5] (in contrast with the "macro" SAT which is typically targeted via direct experimental manipulation) and initial empirical work supported the concept $[6,7]$. Others have noted that individuals are continuously making choices about where to position themselves on the SAT curve in the course of responding [8]. Moreover, in non-experimental work, respondents are potentially making decisions about time due to other pressures (i.e., boredom, fatigue, or testing anxiety may play a role in some settings). This study probes the general utility of the SAT in anticipating behavior in a broad variety of measurement scenarios wherein we study the association of idiosyncratic within-person variation in response time with accuracy.

As response time data is increasingly available for a range of measures, there is also rapid development of a suite of statistical approaches for the study of response time, especially in conjunction with response accuracy $[9,10,4,11,12]$. These approaches account, or don't, for the speed-accuracy tradeoff in several ways. For example, the hierarchical model [9] — which has been widely used in educational measurement settings to model response time behavior for a broad variety of tasks - posits no within-person interplay between speed and accuracy. Other approaches [4] explicitly link response time and accuracy based on models of decision-making (such an approach has experimental support [13]) and still others [11] upweight rapid responses in terms of how they inform inferences about respondent ability. These approaches all make presumptions about interplay between response time and accuracy that may not be empirically supported in specific contexts.

While it is clear that the SAT is a useful hypothesis for describing behavior in some settings, we argue that it deserves further scrutiny when applied to non-experimental data across a range of challenges. The goal of this project is to study, in a variety of data, whether the general intuition behind the SAT holds. Conceptually, this study builds on work suggesting that additional time spent on a response does not always increase its accuracy $[14,15]$. In particular, those projects suggested that increases in time spent on an item were associated with increases in accuracy, but only up to a certain point; in particular, they suggested a curvilinear relationship between response time and accuracy.

We explore this issue using a large number of datasets containing both response accuracy and time from various cognitive tasks. We combine this data with an analytic approach that leverages both an item response model and individual-level variation in response time. We use the item response model to generate an estimate of the probability of accuracy for a person-item interaction. We then use within-person variation in response time to ask if extra time spent on an item tends to yield marginal increases in accuracy net of the probability of accuracy suggested by the item response model. In such cases, the basic logic of the SAT holds. But, of course, it need not.

Alongside this main question, we ask several additional questions pertaining to interplay between speed and accuracy. We focus on issues of interest that have seen relatively limited empirical work (especially across diverse data). We ask whether there is heterogeneity in the association between time usage and accuracy as a function of the challenge (i.e., the prob- 
ability of accuracy as specified by a model for item responses) of the interaction. Turning to items, we ask about the existence of item-level variation in the degree to which marginal changes in time predict change in accuracy. We then ask about the association between person-level speed and accuracy as well as variation in speed. Finally, given the interest in formal models linking time and accuracy, we examine how predictive of response accuracy an individual's speed tends to be in out-of-sample analyses. Collectively, answers to these questions offer novel insight as to what response time data might bring to psychometric models and what types of empirical phenomena may be encountered as more response time data are brought to bear on psychological measures.

\section{Methods}

\section{$2.1 \quad$ Data}

We consider item response datasets containing a variety of tasks and with respondents of various ages; they are documented in the Supplemental Information (SI). The primary criteria for inclusion were: (1) time pressures were not experimentally manipulated across the tasks, ${ }^{1}(2)$ the data came from cognitive tasks, and (3) accuracy can be appropriately modeled as a monotonically increasing function of some latent trait. Data that are appropriately modeled using item response theory (IRT) [16] models with monotonic item response functions would thus be permissible. In contrast, data from measures of affective traits (e.g., personality) or otherwise characterized by non-monotonic models - e.g., "D" models [17] or "unfolding" models [18] — would not be eligible for inclusion in this study. We focus on data that had responses scored in two categories (e.g., correct or incorrect). ${ }^{2}$ Collectively, these data draw from measures that span a range of constructs measured at ages across the lifecourse

Descriptive statistics, including the size of each dataset, are in Table 1. Data range from the relatively small in scale - e.g., 30 people or $<10$ items to the quite large $-50,000$ people and thousands of items. For both design reasons and due to nonresponse, not all individuals attempt all items. In other

\footnotetext{
${ }^{1}$ In some cases (e.g., the Hearts \& Flowers data) time pressure is manipulated across blocks. We examine this variation in the SI but focus here on a single block with constant time pressure. In other cases (e.g., the Reading Fluency and Comp data), the test as a whole was timed but there was not intentional variation of the time pressure across tasks.

${ }^{2}$ In a few cases (e.g., NSHAP), we dichotomized polytomously scored responses so as to increase the number of available items.
}

cases, items are attempted multiple times.

Figure 2 describes response time in these data. Given the skew associated with time, we use logged time throughout. Tests vary substantially in terms of the amount of time required per interaction. Some tests have items that require less than 1s on average while others have items that require more than $1 \mathrm{~m}$. We order the data by mean response time in our presentation of results. There is also variation in the difficulty of the items, as proxied by average percent correct, across the assessments. Some of the tests have items for which only half of the responses are correct while others have items for which responses are nearly always correct. As described below, we attempt to adjust for this via item response models.

\subsection{Analysis}

The approach used here -in particular, combining probabilities from item response models with fixed effects - draws from earlier work [19]. ${ }^{3}$ We have also verified that it behaves as expected via simulation in the context of several different models for the joint distribution of time and accuracy, see SI.

\subsubsection{Mapping speed-accuracy curves}

We first estimate within-person speed-accuracy curves. ${ }^{213}$ To do this, we rely upon estimates of $p_{0}$, the probability of a correct response generated from application of an item response model; specifically, the Rasch model [20]. We estimate

$$
p_{0}=\operatorname{Pr}\left(x_{p i}=1\right)=\sigma\left(\theta_{p}-\delta_{i}\right)
$$

where $\theta_{p}$ and $\delta_{i}$ are person-level and item-level pa- ${ }_{218}$ rameters respectively and $\sigma(x)=(1+\exp (-x))^{-1}$. ${ }_{219}$ Estimation is performed using two approaches. When ${ }_{220}$ a conventional item response matrix can be constructed, 221 we use conventional IRT approaches [21]; when this ${ }_{222}$ is not possible - in particular, when respondents take ${ }_{223}$ multiple attempts at an item -we use random effects 224 model to similar effect [22].

We then use $p_{0}$ in our attempt to model associations between marginal within-person changes in time usage and accuracy. We allow for nonlinear effects in time (i.e., along the lines of those shown in Figure 1) by mapping $\log t$ onto a b-spline basis; we denote this

\footnotetext{
${ }^{3}$ An analytic plan was registered on June 12020 , https: //osf.io/w5u3a. We do not describe this as a preregistration as it was registered following preliminary analysis of some data Further, as described in the SI, we have made some (relatively modest) adjustments to this analytic plan.
} 
as $b(\log t) j .{ }^{4}$ We consider as a baseline model

$$
x_{p i} \sim N\left(\mathrm{~L}\left(b\left(\log t_{p i}\right)_{j}, p_{0, p i}\right)+\lambda_{p}+\gamma_{i}, \sigma_{x}^{2}\right) .
$$

where $\mathrm{L}()$ indicates a linear function of its arguments (e.g., $\mathrm{L}(x, y)=\alpha x+\beta y)$. Note that we rely upon a linear probability model. The fixed effects $\lambda_{p}$ and $\gamma_{i}$ capture person- and item-level features. This model assumes no change in a respondent's speed or ability through the assessment and relies on a relatively constrained model to generate $p_{0}$; we discuss potential limitations stemming from these assumptions below.

\subsubsection{Heterogeneity in SAT curves}

Note that Eqn 2 assumes that changes in accuracy are independent of the challenge of the interaction; a marginal increase in time on an item that is relatively hard for a person is assumed to be as useful as a marginal increase in time on an item that is easy for a person. We now relax this assumption. To explore heterogeneity as a function of $p_{0}$, we then consider

$$
x_{p i} \sim N\left(\operatorname{SL}\left(b\left(\log t_{p i}\right)_{j}, p_{0, p i}\right)+\lambda_{p}+\gamma_{i}, \sigma_{x}^{2}\right) .
$$

where $\mathrm{SL}()$ is a saturated linear function of its arguments (e.g., $\operatorname{SL}(x, y)=\alpha x+\beta y+\eta x y$, with the one caveat that we do not include interaction terms between the splines). We then consider $\frac{\partial f}{\partial \log t}$ where $f$ is the center of the normal density in Eqn 3. The goal is to explicitly identify regions of $\left(p_{0}, t\right)$ space where additional time predicts an increase $\left(\frac{\partial f}{\partial \log t}>0\right)$ or decrease $\left(\frac{\partial f}{\partial \log t}<0\right)$ in accuracy.

\subsubsection{Item- and person-level analyses}

To study the associations of marginal increases in time with accuracy for individual items, we consider the following model separately for each item

$$
x_{p} \sim N\left(\beta_{1} \log \left(t_{p}\right)+\beta_{2} p_{0, p}, \sigma_{x}^{2}\right)
$$

where $p$ indexes all individuals. The estimate of $\beta_{1}$ is an indicator of the marginal association between time and accuracy for each item. To determine whether there is a patterning of this indicator of association with the item's difficulty, we also consider $r\left(\beta_{1}, \delta_{i}\right)$ (with $\delta_{i}$ from Eqn 1).

To study person-level associations between speed and ability (i.e., $\theta$ in Eqn 1), we estimate

$$
\widetilde{\log \left(t_{p i}\right)} \sim N\left(-1 \cdot \tau_{p}, \sigma_{t}^{2}\right)
$$

\footnotetext{
${ }^{4}$ As used here, B-splines are a map from $\mathbb{R}^{1}$ to $\mathbb{R}^{J}$ where $J$ is specified by the user. Illustrations of these maps can be seen in, for example, Figure 5.20 of [23]. To implement this mapping, we use $J=4$ and the defaults in the bs function [24].
}

where $\widetilde{\log \left(t_{p i}\right)}$ represents demeaned (at item-level) re- $\quad{ }_{268}$ sponse times and we additionally assume $\tau_{p} \sim N\left(0, \sigma_{\tau}^{2}\right)$. ${ }_{269}$ We multiply $\tau$ by -1 so that $\tau$ represents speed (i.e., $\quad 270$ a higher $\tau$ will be associated with lower time). We 271 first examine $r\left(\tau_{p}, \theta_{p}\right)$ so as to determine whether 272 higher ability respondents tend to be faster or slower ${ }^{273}$ responders. Motivated by previous observations of 274 within-person variation in speed [25], we then con- 275 sider such variation. Focusing on items with at least 276 100 responses, we find the quantile in the response ${ }_{277}$ time distribution of each response (i.e., the rank) ${ }_{278}$ for a person and take the standard deviation of that ${ }_{279}$ quantity (which we denote $\sigma_{\text {rank }}$ ). ${ }^{5}$ We then consider ${ }_{280}$ $r\left(\theta_{p}, \sigma_{\text {rank }}\right)$ as an indication of whether within-person 281 variation in speed is associated with ability.

\subsubsection{Predictive Accuracy}

283

Finally, we ask about the relative gain in the prediction of accuracy that we get from response time. We do this by comparing the accuracy of predictions in a $10 \%$ hold-out-sample of item responses using models trained in the remaining $90 \% .{ }^{6}$ For this exercise, we first standardize response time within each item. Predictive performance is based on a transformation of the likelihood meant to provide intuition about itemlevel responses; if $\ell$ is the $\log$-likelihood for a response with predicted accuracy of $P$,

$$
\ell_{p i}=x_{p i} \log \left(P_{p i}\right)+\left(1-x_{p i}\right) \log \left(1-P_{p i}\right),
$$

we consider $\exp \left(\overline{\ell_{p i}}\right)$ (where the average is taken over ${ }^{294}$ $p$ and $i$ ).

We consider six alternatives (denoted A-F) for 296 $P_{p i}$. As context for evaluating gains in each dataset, ${ }_{297}$ we first predict (A) using the invariant proportion ${ }_{298}$ of correct responses in each dataset, $P_{p i}=\bar{x}$. We ${ }^{299}$ then consider item-level variation in accuracy and 300 predict based on (B) the proportion correct by item, 301 $P_{p i}=\sum_{p} x_{p i} / n_{p}$ where there are $n_{p}$ responses to item $i$. We now incorporate person-level information using three quantities: the individual's proportion of correct responses, the individual's mean standardized response time, and, due to conceptual [26] and empirical [27] interest in response times for correct responses, the individual's mean standardized response time for correct responses. ${ }^{7}$ For each of these

\footnotetext{
${ }^{5}$ We note one important limitations of this analysis. Data collected in an adaptive fashion leads to potential concentration of respondents into certain items.

${ }^{6}$ Note that we omit both the NWEA and Assistments data from this analysis given the fact that the first data are adaptive and the second data may have dynamics in ability that are poorly captured by our approach.

${ }^{7}$ So as to make comparisons between relatively similar bits of information, we focus on predictions based on quantities
} 
three predictors, $z$, we predict $(\mathrm{C}-\mathrm{E})$ based on fitted logistic regression models containing the item proportion correct and one of the three predictors; i.e., $P_{p i}=\sigma\left(b_{0}+b_{1} \sum_{p} x_{p i} / n_{p}+b_{2} z_{p i}\right)$ where $b_{0}, b_{1}, b_{2}$ are estimated via logistic regression. Finally, we use both time and accuracy information and predict $(\mathrm{F})$ based on both the individual's proportion correct responses and mean standardized response time. Note that outof-sample responses are predicted purely on the basis of in-sample information (i.e., out-of-sample response time is not used)/ We consider analyses that utilize item-level response time (including out-of-sample response time) in the SI. ${ }^{8}$

\section{Results}

\subsection{Mapping the SAT}

Using the approach in Eqn 2, we first consider baseline speed-accuracy curves. Results are in Figure 3. Each panel in that figure has a similar form; they are also similar to the format of Figure 1 . The $\mathrm{x}$ axis captures time spent on the item. ${ }^{9}$ The y-axis shows changes to the estimated accuracy net of $p_{0}$. The densities show the distribution of $\log (t)$ for the data split by correct/incorrect responses. The curves shows estimated changes in accuracy as a function of time; recall that the SAT would suggest that such lines be monotonically increasing as longer responses are associated with increases in accuracy. Results are also categorized by age (line color).

We readily observe a large variety of behavior in terms of the within-person relationship between response time and accuracy. In some cases (e.g., Lexical, Arithmetic), longer response times do generally translate into increased accuracy. However, this is not universally true. In come cases (e.g., working memory, NSHAP), longer time is uniformly associated with a decline in accuracy. In other cases (e.g., rotation, reading fluency), associations with accuracy for additional response time can be positive or negative. While these results suggest that a wide variety of relationships are possible, we emphasize two points of consistency.

Note the role of time limits. Consider, for example, the Hearts Flowers and Rotation tasks. For those, we observe steep declines in accuracy as a function of time increases when response times are near

computed in relatively comparable manners instead of focusing on, for example, the IRT-based probability $p_{0}$

${ }^{8}$ The analyses presented in the SI are the ones proposed in the original registration.

${ }^{9}$ We focus here on $\log t$ but results are similar when we consider results in seconds, see SI. their maximum. In these cases, we hypothesize that respondents began to choose answers with less certainty when they neared the time limit for each task. Note that we also detect a relative increase in the density of incorrect responses prior to the time limit for these two datasets. We further illustrate the role of time limits along the lines described here using variation in time pressure in additional data from the Hearts Flowers task, see SI.

Within age, we generally observe variation in curve shape. However, if we focus on older respondents (the HRS and NSHAP data), we observe strong negative slopes. In the context of these data, we hypothesize that the nature of the curve is due in part to both the age of the respondent and the type of task in these data. We further investigated this possibility using the PIAAC data, see SI; this analysis supports the supposition that the nature of the HRS and NSHAP tasks play some role (it does not seem to be simply the age of the respondent).

Figure 3 focuses on associations between response time and accuracy net of the underlying challenge (i.e., $p_{0}$ ) of the interaction. We now ask whether there may be heterogeneous effects associated with interplay between speed and accuracy as a function of this challenge. We do so by constructing curves similar to the ones shown in Figure 3 but that vary by the challenge of the interaction. Rather than focusing on the curve, we focus on the curve's instantaneous slope (i.e., $\frac{\partial f}{\partial \log t}$ ).

\subsection{Heterogeneity as a function of $p_{0}$}

We now allow for heterogeneity as a function of the interaction's $p_{0}$. Conceptually, this is equivalent to asking if the shape of the curve shown in Figure 1 is sensitive to the value of $p_{0}$ (i.e., the location of the horizontal gray line). Results based on the approach in Eqn 3 are shown in Figure 4. In this figure (as in Figure 3), the x-axis shows response time for the test. The y-axis shows the $p_{0}$ of the interaction; a value of, for example, 0.7 means that an individual responding to a given item is projected by the Rasch model to have a $70 \%$ probability of getting the item correct. At a given point in each panel of the figure, the color represents $\frac{\partial f}{\partial \log t}$. Areas in blue correspond to $\frac{\partial f}{\partial \log t}>0$ suggesting that a marginal increase in time for an interaction of the given challenge will be positive (i.e., the SAT seems to be operant). Areas in red correspond to $\frac{\partial f}{\partial \log t}<0$; in such areas, marginal increases in time are associated with decreases in accuracy. If we consider a vertical strip, a change in color suggests sensitivity in the time/accuracy relationship as a function of $p_{0}$. Likewise, when we consider a hor- 
izontal strip a change in color suggests sensitivity in the time/accuracy relationship to the baseline duration of the response.

We start with the datasets consisting of rapid tasks. Results are fairly heterogeneous. One fairly universal finding (Rotation and Set being exceptions) is that, across values of $p_{0}$, shorter responses are those that are likely to benefit from some increase in accuracy if they are marginally longer (i.e., the left side of each panel tends to be blue); this is perhaps due to marginally longer responses being less due to rapid guessing. The boundary between blue and red also tends to slope from upper left to bottom right such that, for a constant response time, marginal increases are more likely to be in the blue as opposed to the red if they represent more challenging interactions. Consider the Add Subtract dataset. If $\log (t)=1.8$ and $p_{0} \approx 0.5$, we observe $\frac{\partial f}{\partial \log t}>0$ while if $p_{0} \approx 0.8$ we observe $\frac{\partial f}{\partial \log t}<0$

With less rapid tasks, many of the same patterns appear. In particular, we observe larger blue regions on the left and boundaries between blue and red regions tend to be negatively sloped. However, there are also cases where the partial derivative is uniformly positive (e.g., PIAAC) or negative (e.g., HRS). All told, these analyses suggest that whether the SAT holds may vary both across the nature of the task but also as a function of the precise conditions within the set of tasks in a given dataset.

\subsection{Item-level heterogeneity}

Using a modified approach (e.g., Eqn 4), we focus on SAT curves for individual items. We focus on the marginal effect of time net of $p_{0}$. Results are shown in Table 2 focusing on only those items that have at least 100 responses. Given that each dataset contained numerous items, we identified those items showing positive/negative marginal associations with time based on estimates of $\beta_{1}$ that were significant after adjusting (via Bonferonni correction) for multiple testing of all items within dataset.

In general, associations tended to be positive or null. However, note that, for example, the chess data had a relatively large proportion of items show a negative association and nearly all data had at least some items that showed negative associations; we speculate on the reasons for such negative associations in the Discussion. We also investigated correlations between item difficulty and the marginal time/accuracy associations. Such associations varied widely across the datasets.

\subsection{Person-level heterogeneity}

We next analyze person-level speed via Eqn 5. Results are shown in Table 3. We first consider correlations between estimates of ability and speed. Correlations vary widely. In some cases, more able respondents are also faster (e.g., chess) in other cases, the opposite is true (e.g., the PIAAC and PISA).

We next consider within-person variation in speed during the test. We observed variation in speed - as indexed by changes in a respondent's rank ordering of response time across items - that was fairly consistent across all the datasets although the ECLS Flanker tasks showed the least amount of withinperson variation. This quantity has an interesting pattern of association with ability. Across nearly all datasets (Lexical being the exception), respondents with larger estimates of $\theta$ showed less variation in speed. Although this association was not always significant, we think it suggestive of a potentially important insight regarding fluctuations in respondent speed and resulting estimates of ability based on the collected responses.

\subsection{Predictive power of response time}

Finally, we examine the predictive power of response time as compared to alternative predictors. Recall that out-of-sample fit is evaluated via $\exp \left(\overline{\ell_{p i}}\right)$ where $\ell_{p i}$ is as in Eqn 6. Results are shown in Figure 5. We focus here on three comparisons (denoted via letters in Figure 5 legend), how prediction changes when we: exchange person-level response accuracy for personlevel response time (C versus $\mathrm{D})$, exchange response time information for response time based only on correct items (D versus E), and combine accuracy and response time information ( $\mathrm{F}$ versus $\mathrm{C} / \mathrm{D}$ ).

With respect to the first comparison (C versus $\mathrm{D})$, we generally make better predictions based on accuracy rather than response time. There are exceptions (ECLS Flanker, Set, Add Subtract, Working Memory, and Mult Div); we emphasize that, especially for data containing more complex tasks that take longer than 10 s, we are better able to predict novel responses using accuracy rather than response time. With respect to the second comparison (D versus $\mathrm{E}$ ), differences were quite small. In only two cases were differences larger than 0.01; in both cases (Groupitizing and MITRE-ETS), prediction was superior when using all response time information. With respect to the third comparison ( $\mathrm{F}$ versus $\mathrm{C} / \mathrm{D}$ ), we generally find that prediction using both response time and accuracy is generally inferior to models based on just a single predictor (response time or accuracy). Similarly, results from analyses in the SI suggest that 
using response time from an individual item response tend to degrade prediction as compared to predicting based on $p_{0}$ alone. In sum, these analyses suggest that response time may not be a useful predictor of behavior in many cases. This could be due, in part, to the fact that additional time on an item may predict both positive and negative changes in accuracy (i.e., Figure 3).

\section{Discussion}

We use the standardized analysis of 29 item response datasets that also contain information on response time to study interplay between speed and accuracy in non-experimental settings. Results suggest that, in these non-experimental settings, marginal increases in time do not necessarily lead to increased accuracy. In some cases, we observed patterns consistent with those predicted by the SAT but, in other cases, we did not. Accuracy either declined or showed an inconsistent relationship with increased response times. Further, there may be additional heterogeneity within a set of tasks when we stratify by the underlying challenge (i.e., $p_{0}$ ) of the interaction. We emphasize that our analytic approach returned appropriate results when data were generated under a variety of joint models for speed and accuracy (see SI) thus offering additional credence to these results.

When we consider associations between time and accuracy at the item-level, we identify items that have both the relationship between those two quantities anticipated by the SAT as well as the opposite. Turning to respondents, we observe inconsistent relationships between respondent speed and ability. While faster respondents are not necessarily more able, we do observe a consistent relationship between variation in respondent speed across items and their ability as respondents with more variation in speed tend to be lower ability. Finally, our predictive analyses suggested that, in general, response time either at the level of individual responses or aggregated across a person's responses - is rarely a strong predictor of accuracy.

We first discuss implications for the SAT. Substantial experimental evidence [1] suggests that artificial manipulation of time pressure has an effect on accuracy. We observe something similar in responses occurring near a time limit. With such data, responses near the time limit tend to be incorrect when the individual spends additional time on the item. These observations are consistent with predictions of the SAT. Our findings suggest that other factors may be at work in observational data and gener- ally tend to reduce the role of the SAT as a plausible first-order explanation for observed behavior.

One substantively interesting case wherein the SAT does not hold involves older respondents (i.e., the HRS, NSHAP). In these data, we observe decreases in accuracy when respondents spend more time on items. We suspect that this finding has to do with both the nature of cognition in older respondents and the tasks in question. With respect to the age of the respondents, they may be experiencing some form of "cognitive aging" - an age-related decline in cognitive functioning [28]. For respondents experiencing cognitive aging, it is possible that a within-person reduction in response speed isn't associated with deliberation and increased accuracy but, rather, confusion and decreased accuracy. Our findings can be read alongside others suggesting a change in the SAT $[29,1]$ as respondents age.

We do not observe consistent evidence that more accurate respondents are generally faster or slower. This could be due to heterogeneity across tasks or, for example, motivational gradients across the datasets. But, when we consider variation in speed, higher ability respondents generally tend to vary less (i.e., they show less fluctuation in their place in the response time distribution item-to-item). Such variation in speed could be a phenotype worth further study. Previous work suggests, for example, that such variation tends to predict cognitive aging in older samples [30].

Although the heterogeneous tasks here may be classified using existing taxa [31], we suspect that our findings could also be used to devise new taxa. For example, various data-working memory, HRS, Chess, NSHAP - show downwardly sloping curves in Figure 3 absent any time limits. This might reflect some underlying similarity to the cognitive processes brought to bear in answering these tasks. Future work could potentially use alternative research modalities (e.g., eye-tracking or imaging studies) to probe whether this may be the case.

Turning now to the utility of incorporating response time into models meant to predict response behavior, we generally find that response time is of limited predictive value. While there may be cases where response-time information provides some increase in predictive accuracy, we generally find response time to be less useful than accuracy in predicting out-of-sample responses. This is consistent with findings in Figure 3 suggesting that the curve of association between time and accuracy is either relatively flat or otherwise not monotonic in many cases. That said, we note that our work does not suggest that response time is not predictive of future behavior or functioning (i.e., events some extended time 
from the point of observation rather than responses collected basically contemporaneously).

We acknowledge limitations. Other features of data collection may be relevant; we discuss a few specific features that may be worth further consideration. We have not addressed, for example, ordering effects [32]. In many cases, items later in the test may appear harder than they would if presented earlier in the test. This may be due, in part, to systematic changes in response time devoted to such items [19]. In analyses of responses collected relatively early versus relatively late in the NWEA testing do suggest differences in the relationship between speed and accuracy (see SI). There are presumably motivational differences across the datasets that we do not measure and cannot study. There is evidence to suggest that emotional states - e.g., worry [33] — that may vary as a function of motivational differences and/or testing pressure may affect the SAT.

There are also potential limitations related to our analytic approach. In particular, the Rasch model that we use may be inadequate for characterizing the relevant item response functions; this may induce bias in, for example, Figure 4 if estimates of $p_{0}$ are distorted. Future work could investigate whether findings can be refined using more alternative item response models. Further, there are also cases where our ability to identify items (e.g., working memory) is relatively weak in the sense that we are classifying a relatively broad class of tasks as a single item. In other cases (e.g., Assistments), the assumption of a static ability may be inappropriate. We think that the potential insights from a common analysis applied to a broad variety of datasets offers great value in spite of these limitations but encourage others to keep these limitations in mind when interpreting our results.

Alongside the above arguments made regarding our substantive understanding of the SAT, our findings have implications for both psychometrics and survey design. For psychometrics, we think there are two principle implications. First, the SAT may have limited utility to describe response behaviors in nonexperimental settings when time pressures are light. Second, response time may offer only limited predictive power in many empirical settings; incorporation of response time into such models - especially in cases where additional time sometimes predicts higher levels of accuracy but other times lesser - needs to be done with care. However, we emphasize that withinperson variation in speed may be a useful phenomena to investigate further; across our data, respondents that showed more variation in speed tended to perform worse. In general, while we agree with others that RT may be used to better inform validity studies [34], we think that a richer empirical grounding on how RT should be expected to behave will be useful in this endeavor; this study is an attempt to provide such grounding.

For survey design, we flag two insights in particular that merit consideration. First, time limits on items should be used with caution. They largely served to increase the number of incorrect responses. If time pressure is not an inherent part of the construct, perhaps time limits need not be utilized? ${ }^{10}$ Second, we note the following question raised by our data: why do some items have relationships with times such that marginal increases by a respondent are associated with decreased accuracy? There are conceptual reasons to suspect that items may have this property. Items that are quite simple - consider either the question of today's date (in the HRS) or a simple arithmetic problem such as $2+2$ in the context of either the Arithmetic or Add Subtract datamay demonstrate this behavior as respondents simply know the answer or do not and longer responses simply indicate befuddlement. But, in general, we suspect there are occasions when such findings suggest poor psychometric performance of the item; for example, some items could be confusing for reasons unrelated to the construct of interest and this could potentially impact the SAT [36]. To diagnose such cases, we would recommend item fit analyses - for example, infit and outfit statistics [37] in the case of the Rasch model - and, when possible, analyses of distractors [38, 39].

In this paper, we consider results from a standard analysis applied to a heterogenous set of cognitive tasks. The results, especially those in Figure 3, are themselves heterogeneous but suggest that there are many occasions wherein additional response time is associated with a decrease in accuracy. We argue that this suggests a need to reconsider whether the SAT is a viable first-order descriptor of behavior in response time data not explicitly manipulated with respect to time pressure. In observational settings, people vary their speed for a variety of reasons (fatigue, boredom, confusion about a specific problem, etc) that diverge from the reasons that people vary their speed in the context of experimental SAT studies. When one experimentally manipulates time pressure, one observes the SAT. However, absent that, people are making decisions that affect speed and accuracy for lots of reasons, not all of which lead to results anticipated

\footnotetext{
${ }^{10}$ This consideration and the subsequent conceptualization of a measure as being either one of "speed" or "power" is an old one (see Ch 17 of [35]) that we find to be continually relevant here.
} 
by the SAT.

\section{References}

[1] Richard P Heitz. The speed-accuracy tradeoff: history, physiology, methodology, and behavior. Frontiers in neuroscience, 8:150, 2014.

[2] Rafal Bogacz, Eric-Jan Wagenmakers, Birte U Forstmann, and Sander Nieuwenhuis. The neural basis of the speed-accuracy tradeoff. Trends in neurosciences, 33(1):10-16, 2010.

[3] Vincent Van Veen, Marie K Krug, and Cameron S Carter. The neural and computational basis of controlled speed-accuracy tradeoff during task performance. Journal of Cognitive Neuroscience, 20(11):1952-1965, 2008.

[4] Roger Ratcliff, Philip L Smith, Scott D Brown, and Gail McKoon. Diffusion decision model: Current issues and history. Trends in cognitive sciences, 20(4):260-281, 2016.

[5] Ian Dennis and Jonathan St BT Evans. The speed-error trade-off problem in psychometric testing. British Journal of Psychology, 87(1):105-129, 1996.

[6] Joseph S Lappin and Kenneth Disch. The latency operating characteristic: Ii. effects of visual stimulus intensity on choice reaction time. Journal of Experimental Psychology, 93(2):367, 1972.

[7] JF Schouten and JAM Bekker. Reaction time and accuracy. Acta psychologica, 27:143-153, 1967.

[8] Gunter Maris and Han Van der Maas. Speedaccuracy response models: Scoring rules based on response time and accuracy. Psychometrika, 77(4):615-633, 2012.

[9] Wim J van der Linden. A hierarchical framework for modeling speed and accuracy on test items. Psychometrika, 72(3):287, 2007.

[10] Jochen Ranger, Jörg-Tobias Kuhn, and JoséLuis Gaviria. A race model for responses and response times in tests. Psychometrika, 80(3):791$810,2015$.

[11] Peter W van Rijn and Usama S Ali. A generalized speed-accuracy response model for dichotomous items. Psychometrika, 83(1):109131, 2018.
[12] Dylan Molenaar, Maria Bolsinova, and Jeroen K Vermunt. A semi-parametric within-subject mixture approach to the analyses of responses and response times. British Journal of Mathematical and Statistical Psychology, 71(2):205228,2018 .

[13] John Palmer, Alexander C Huk, and Michael N Shadlen. The effect of stimulus strength on the speed and accuracy of a perceptual decision. Journal of vision, 5(5):1-1, 2005.

[14] Maria Bolsinova and Dylan Molenaar. Modeling nonlinear conditional dependence between response time and accuracy. Frontiers in psychology, 9:1525, 2018.

[15] Haiqin Chen, Paul De Boeck, Matthew Grady, Chien-Lin Yang, and David Waldschmidt. Curvilinear dependency of response accuracy on response time in cognitive tests. Intelligence, 69:16-23, 2018.

[16] Wim J van der Linden and Ronald K Hambleton. Handbook of modern item response theory. Springer Science \& Business Media, 2013.

[17] Dylan Molenaar, Francis Tuerlinckx, Han LJ van der Maas, et al. Fitting diffusion item response theory models for responses and response times using the r package diffirt. Journal of Statistical Software, 66(4):1-34, 2015.

[18] James S Roberts, John R Donoghue, and James E Laughlin. A general item response theory model for unfolding unidimensional polytomous responses. Applied Psychological Measurement, 24(1):3-32, 2000.

[19] Ben Domingue, Klint Kanopka, Ben Stenhaug, Jim Soland, Megan Kuhfeld, Steve Wise, and Chris Piech. Interplay between speed and accuracy: Novel empirical insights based on $1 / 4$ billion item responses. PsyArXiv, 2020.

[20] Georg Rasch. Probabilistic models for some intelligence and attainment tests. ERIC, 1993.

[21] R Philip Chalmers et al. mirt: A multidimen- ${ }^{802}$ sional item response theory package for the $\mathrm{r}{ }_{803}$ environment. Journal of Statistical Software, ${ }^{804}$ 48(6):1-29, 2012.

[22] Paul De Boeck, Marjan Bakker, Robert Zwitser, ${ }_{806}$ Michel Nivard, Abe Hofman, Francis Tuerlinckx, $\quad{ }_{807}$ Ivailo Partchev, et al. The estimation of item re- ${ }_{808}$ sponse models with the lmer function from the 809 lme4 package in r. Journal of Statistical Soft- ${ }_{810}$ ware, 39(12):1-28, 2011. 
[23] Jerome Friedman, Trevor Hastie, and Robert Tibshirani. The elements of statistical learning, volume 1. Springer series in statistics New York, 2001.

[24] R Core Team. R: A Language and Environment for Statistical Computing. R Foundation for Statistical Computing, Vienna, Austria, 2019.

[25] Steven L Wise. Response time as an indicator of test taker speed: Assumptions meet reality. Measurement: Interdisciplinary Research and Perspectives, 13(3-4):186-188, 2015.

[26] Matthew C Davidson, Dima Amso, Loren Cruess Anderson, and Adele Diamond. Development of cognitive control and executive functions from 4 to 13 years: Evidence from manipulations of memory, inhibition, and task switching. Neuropsychologia, 44(11):2037-2078, 2006.

[27] Shiyang Su and Mark L Davison. Improving the predictive validity of reading comprehension using response times of correct item responses. $A p$ plied Measurement in Education, 32(2):166-182, 2019.

[28] Elliot M Tucker-Drob. Cognitive aging and dementia: A life-span perspective. Annual Review of Developmental Psychology, 1:177-196, 2019.

[29] Timothy A Salthouse. Adult age and the speedaccuracy trade-off. Ergonomics, 22(7):811-821, 1979.

[30] Martin Lövdén, Shu-Chen Li, Yee Lee Shing, and Ulman Lindenberger. Within-person trialto-trial variability precedes and predicts cognitive decline in old and very old age: Longitudinal data from the berlin aging study. Neuropsychologia, 45(12):2827-2838, 2007.

[31] Kevin S McGrew. Chc theory and the human cognitive abilities project: Standing on the shoulders of the giants of psychometric intelligence research. Intelligence, 37:1-10, 2009.

[32] Dries Debeer and Rianne Janssen. Modeling item-position effects within an irt framework. Journal of Educational Measurement, 50(2):164185, 2013.

[33] Lauren S Hallion, Susan N Kusmierski, and M Kathleen Caulfield. Worry alters speedaccuracy tradeoffs but does not impair sustained attention. Behaviour Research and Therapy, page 103597, 2020.
[34] Zhi Li, Jayanti Banerjee, and Bruno D Zumbo. Response time data as validity evidence: Has it lived up to its promise and, if not, what would it take to do so. In Understanding and investigating response processes in validation research, pages 159-177. Springer, 2017.

[35] Harold Gulliksen. Theory of mental tests. Lawerence Erlbaum Associates, 1950.

[36] Kobe Desender, Annika Boldt, Tom Verguts, ${ }^{867}$ and Tobias H Donner. Confidence predicts ${ }_{868}$ speed-accuracy tradeoff for subsequent decisions. $\quad 869$ Elife, 8:e43499, 2019.

[37] Margaret Wu and Raymond J Adams. Proper- ${ }^{871}$ ties of rasch residual fit statistics. Journal of ${ }_{872}$ Applied Measurement, 2013.

[38] David Thissen, Lynne Steinberg, and Anne R 874 Fitzpatrick. Multiple-choice models: The dis- 875 tractors are also part of the item. Journal of ${ }_{876}$ Educational Measurement, 26(2):161-176, 1989. 877

[39] Han LJ Van der Maas and Enkhbold Nyam- 878 suren. Cognitive analysis of educational games: $\quad 879$ The number game. Topics in cognitive science, 880 9(2):395-412, 2017.

\section{Acknowledgements}

This work was supported in part by the Institute of ${ }_{883}$ Education Sciences (R305B140009) and a gift from ${ }_{884}$ an anonymous donor. The HRS (Health and Retire- 885 ment Study) is sponsored by the National Institute 886 on Aging (grant number NIA U01AG009740) and is ${ }_{887}$ conducted by the University of Michigan.

\section{9 89 81} 860 861 862 863 65 66 864 870 (n) . 然 (1) m .

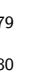


Table 1: Descriptive statistics for the datasets (including time limits for those datasets that impose them at the item level).

\begin{tabular}{lrrrc}
\hline & \# people & \# items & \# Interactions & Time Limit (s) \\
\hline Lexical & 93 & 15 & 66059 & \\
RR98 Accuracy & 30 & 33 & 12194 & \\
Hearts Flowers & 255 & 8 & 5071 & 1.5 \\
LDT & 104 & 495 & 51480 & \\
ECLS Flanker & 12008 & 20 & 239963 & 10.0 \\
ECLS DCCS & 12023 & 30 & 360430 & 10.0 \\
Motion & 106 & 30 & 31778 & 10.0 \\
MSIT & 740 & 24 & 16739 & 2.5 \\
Reading Fluency & 3943 & 315 & 212507 & \\
Reading Comp & 3947 & 448 & 165630 & \\
Arithmetic & 895 & 173 & 133796 & \\
Groupitizing & 481 & 88 & 40450 & \\
Rotation & 95 & 10 & 950 & 7.5 \\
Set & 355 & 10 & 3550 & 20.0 \\
Letter Chaos & 233 & 10 & 2330 & 20.0 \\
Add Subtract & 16190 & 60 & 200297 & \\
Working Memory & 194 & 4 & 1365 & \\
Mult Div & 14184 & 60 & 174517 & 20.0 \\
HRS & 2215 & 20 & 36785 & \\
Chess & 258 & 80 & 19135 & 30.0 \\
PISA Reading & 42398 & 223 & 1850217 & \\
PERC & 1680 & 15 & 25132 & \\
MITRE-ETS & 801 & 95 & 75912 & 90.0 \\
Assistments & 2306 & 3518 & 131864 & \\
NSHAP & 2210 & 13 & 28717 & \\
PIAAC & 2278 & 104 & 55563 & \\
PISA Math & 21995 & 60 & 323887 & \\
NWEA Grade 3 & 49998 & 5181 & 1952749 & \\
NWEA Grade 8 & 49984 & 6049 & 1888845 & \\
\hline
\end{tabular}


Table 2: Item-level analysis for those items with $>100$ responses. The percentage of items showing positive or negative coefficients of $\log (t)$ predicting accuracy (e.g., estimates of $\beta_{1}$ from Eqn 4) are those that remain after Bonferonni correction. Only significant correlations between difficulty and $\beta_{1}$ are shown.

\begin{tabular}{lcccccc}
\hline & $\mathrm{N}$ items & $\%\left(\beta_{1}>0\right)$ & $\%\left(\beta_{1}<0\right)$ & $r\left(\beta_{1}, \delta_{i}\right)$ & $\mathrm{CI}-\mathrm{L}$ & $\mathrm{CI}-\mathrm{U}$ \\
\hline Lexical & 15 & 40 & 13 & 0.22 & -0.33 & 0.66 \\
RR98 Accuracy & 32 & 0 & 0 & -0.11 & -0.44 & 0.25 \\
Hearts Flowers & 8 & 12 & 12 & 0.86 & 0.39 & 0.97 \\
LDT & 495 & 1 & 0 & -0.14 & -0.22 & -0.05 \\
ECLS Flanker & 20 & 70 & 10 & 0.78 & 0.52 & 0.91 \\
ECLS DCCS & 30 & 40 & 0 & 0.87 & 0.74 & 0.94 \\
Motion & 30 & 13 & 10 & -0.48 & -0.71 & -0.14 \\
MSIT & 24 & 50 & 0 & 0.70 & 0.41 & 0.86 \\
Reading Fluency & 292 & 10 & 4 & -0.13 & -0.24 & -0.01 \\
Reading Comp & 408 & 11 & 2 & -0.40 & -0.48 & -0.32 \\
Arithmetic & 170 & 31 & 1 & 0.25 & 0.11 & 0.39 \\
Groupitizing & 88 & 59 & 0 & 0.29 & 0.08 & 0.47 \\
Rotation & 10 & 0 & 0 & -0.04 & -0.65 & 0.61 \\
Set & 10 & 0 & 80 & -0.41 & -0.82 & 0.30 \\
Letter Chaos & 10 & 20 & 0 & 0.30 & -0.40 & 0.78 \\
Add Subtract & 60 & 38 & 7 & -0.11 & -0.35 & 0.15 \\
Working Memory & 4 & 0 & 75 & 0.83 & -0.64 & 1.00 \\
Mult Div & 60 & 3 & 63 & -0.05 & -0.30 & 0.20 \\
HRS & 20 & 5 & 65 & 0.17 & -0.30 & 0.57 \\
Chess & 80 & 5 & 26 & -0.03 & -0.25 & 0.19 \\
PISA Reading & 218 & 39 & 16 & -0.25 & -0.37 & -0.12 \\
PERC & 15 & 13 & 40 & -0.26 & -0.68 & 0.29 \\
MITRE-ETS & 95 & 13 & 2 & -0.63 & -0.73 & -0.49 \\
Assistments & 604 & 0 & 1 & 0.10 & 0.02 & 0.18 \\
NSHAP & 13 & 8 & 54 & 0.21 & -0.38 & 0.68 \\
PIAAC & 104 & 71 & 0 & 0.53 & 0.37 & 0.65 \\
PISA Math & 60 & 28 & 15 & 0.05 & -0.20 & 0.30 \\
NWEA Grade 3 & 3694 & 3 & 0 & -0.09 & -0.13 & -0.06 \\
NWEA Grade 8 & 3331 & 3 & 2 & -0.02 & -0.06 & 0.01 \\
\hline
\end{tabular}


Table 3: Person-level associations between ability $(\theta)$, speed $(\tau)$, and variation in speed $\left(\sigma_{\text {rank }}\right)$.

\begin{tabular}{lccccc}
\hline & $r(\theta, \tau)$ & $\mathbb{E}\left(\sigma_{\text {rank }}\right)$ & $r\left(\theta, \sigma_{\text {rank }}\right)$ & CI-L & CI-U \\
\hline Lexical & 0.15 & 0.25 & 0.06 & -0.14 & 0.26 \\
RR98 Accuracy & 0.17 & 0.25 & -0.14 & -0.47 & 0.24 \\
Hearts Flowers & -0.20 & 0.25 & -0.36 & -0.46 & -0.25 \\
LDT & -0.05 & 0.22 & -0.61 & -0.72 & -0.47 \\
ECLS Flanker & -0.05 & 0.19 & -0.18 & -0.20 & -0.16 \\
ECLS DCCS & -0.11 & 0.23 & -0.22 & -0.24 & -0.20 \\
Motion & 0.06 & 0.26 & -0.43 & -0.57 & -0.26 \\
MSIT & -0.23 & 0.24 & -0.27 & -0.34 & -0.20 \\
Reading Fluency & -0.03 & 0.21 & -0.12 & -0.15 & -0.09 \\
Reading Comp & -0.28 & 0.22 & -0.20 & -0.23 & -0.17 \\
Arithmetic & 0.19 & 0.22 & -0.56 & -0.61 & -0.52 \\
Groupitizing & -0.46 & 0.24 & -0.41 & -0.48 & -0.33 \\
Rotation & 0.12 & 0.22 & -0.05 & -0.25 & 0.15 \\
Set & 0.16 & 0.25 & -0.11 & -0.21 & -0.01 \\
Letter Chaos & -0.08 & 0.22 & -0.20 & -0.32 & -0.07 \\
Add Subtract & 0.08 & 0.23 & -0.13 & -0.14 & -0.11 \\
Working Memory & 0.31 & 0.23 & -0.08 & -0.22 & 0.06 \\
Mult Div & 0.05 & 0.24 & -0.13 & -0.15 & -0.11 \\
HRS & 0.41 & 0.24 & -0.06 & -0.10 & -0.02 \\
Chess & 0.44 & 0.24 & -0.01 & -0.13 & 0.11 \\
PISA Reading & -0.23 & 0.25 & -0.28 & -0.29 & -0.27 \\
PERC & -0.43 & 0.24 & -0.20 & -0.24 & -0.15 \\
MITRE-ETS & -0.62 & 0.22 & -0.23 & -0.29 & -0.16 \\
Assistments & -0.36 & 0.24 & -0.27 & -0.31 & -0.23 \\
NSHAP & 0.30 & 0.24 & -0.07 & -0.11 & -0.03 \\
PIAAC & -0.33 & 0.23 & -0.48 & -0.51 & -0.45 \\
PISA Math & -0.15 & 0.24 & -0.06 & -0.08 & -0.05 \\
NWEA Grade 3 & 0.02 & 0.24 & -0.14 & -0.15 & -0.14 \\
NWEA Grade 8 & 0.04 & 0.23 & -0.21 & -0.22 & -0.20 \\
\hline
\end{tabular}


Figure 1: Prototypical speed-accuracy curve. For an individual, increases in response time are, all else equal, expected to translate into increase in accuracy relative to expectation (gray line); this is indicated by the upward slope of the blue line. Note that there is no time limit considered in this hypothetical scenario.

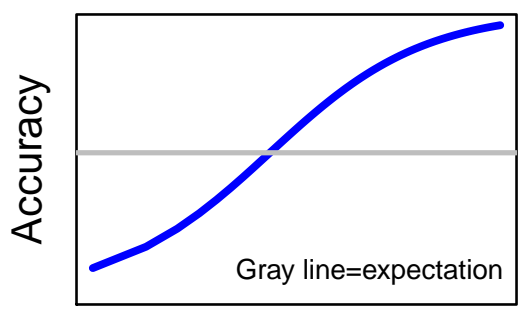

$\log t$

Figure 2: Response Time. Left: Boxplots of response time (logged) for each of the datsets. Right: Comparison of mean item-level accuracy (x-axis) and response time (y-axis) across the items. Horizontal lines show $1 \mathrm{~s}, 10 \mathrm{~s}$, and $1 \mathrm{~m}$ increments.
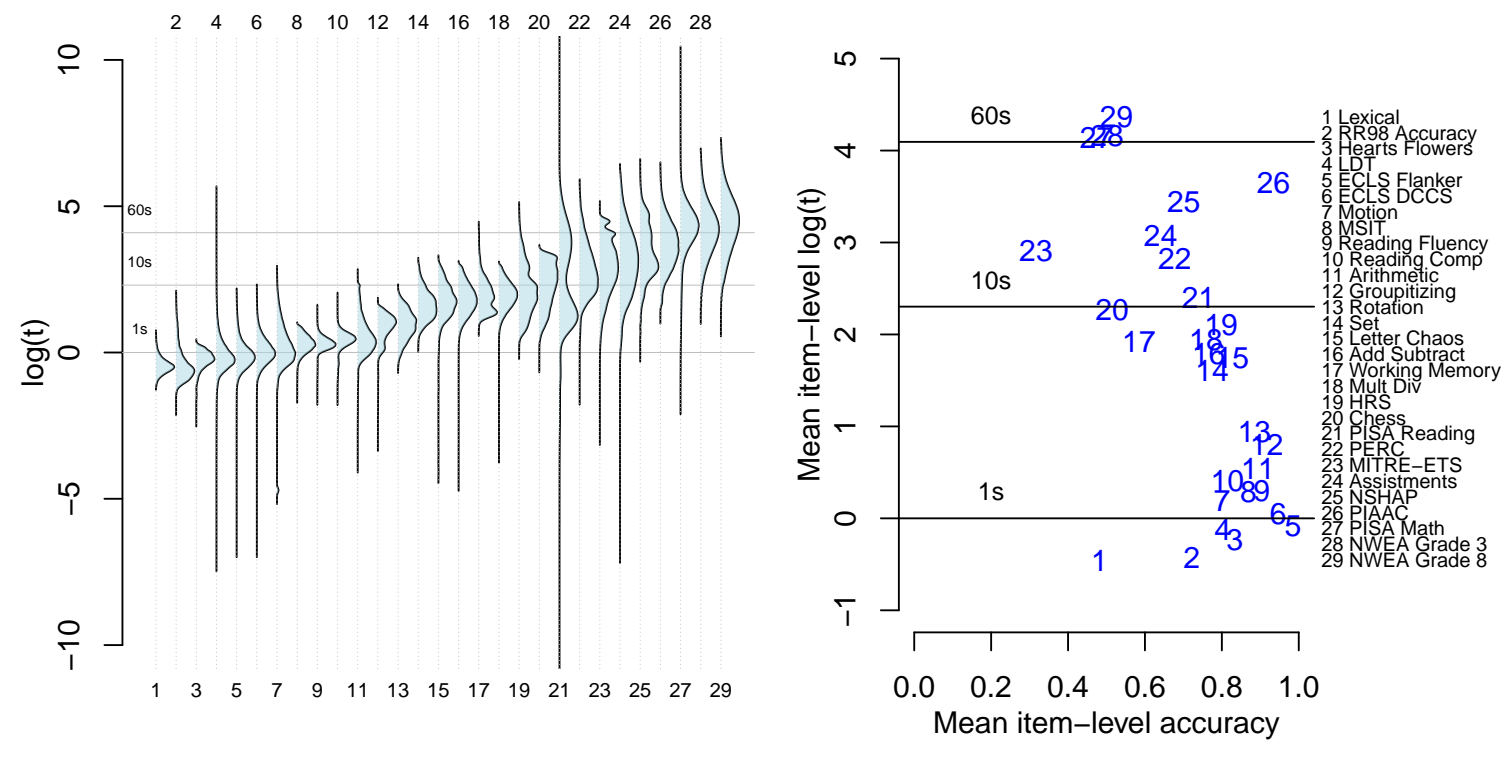
Figure 3: Estimated association between response time and changes to accuracy (net of $p_{0}$ ). For each test, the x-axis spans from the .1 to .9 quantiles of observed $\log (t)$. The $y$-axis focuses on offsets to the test mean of (IRT-based) $\operatorname{Pr}(x=1)$. Curves represent estimated accuracy as a function of time (colored by respondent age). Densities at bottom of panel show distribution of response times for each test separately by response type. Vertical lines represent time limits (where applicable).
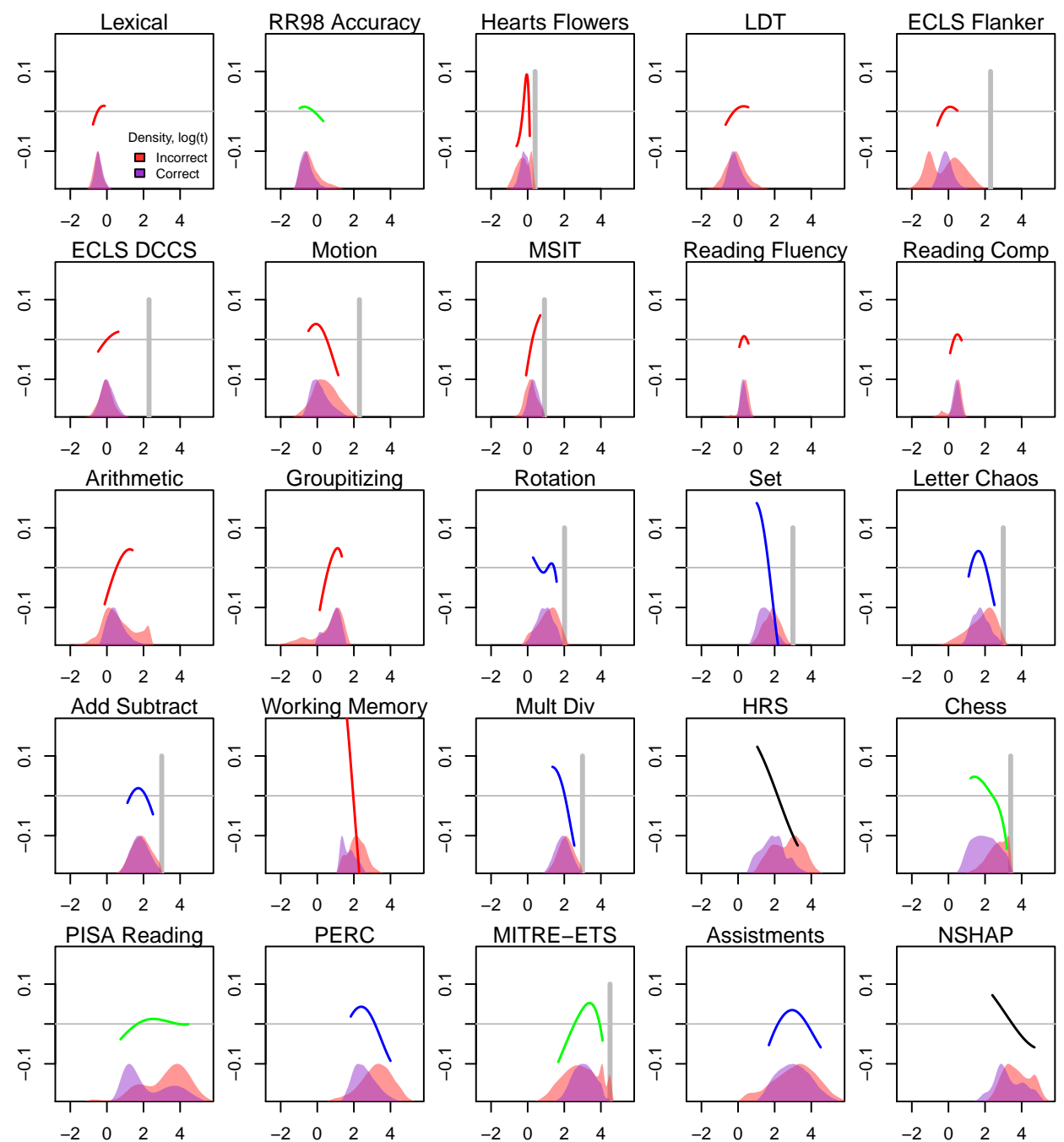

NSHAP
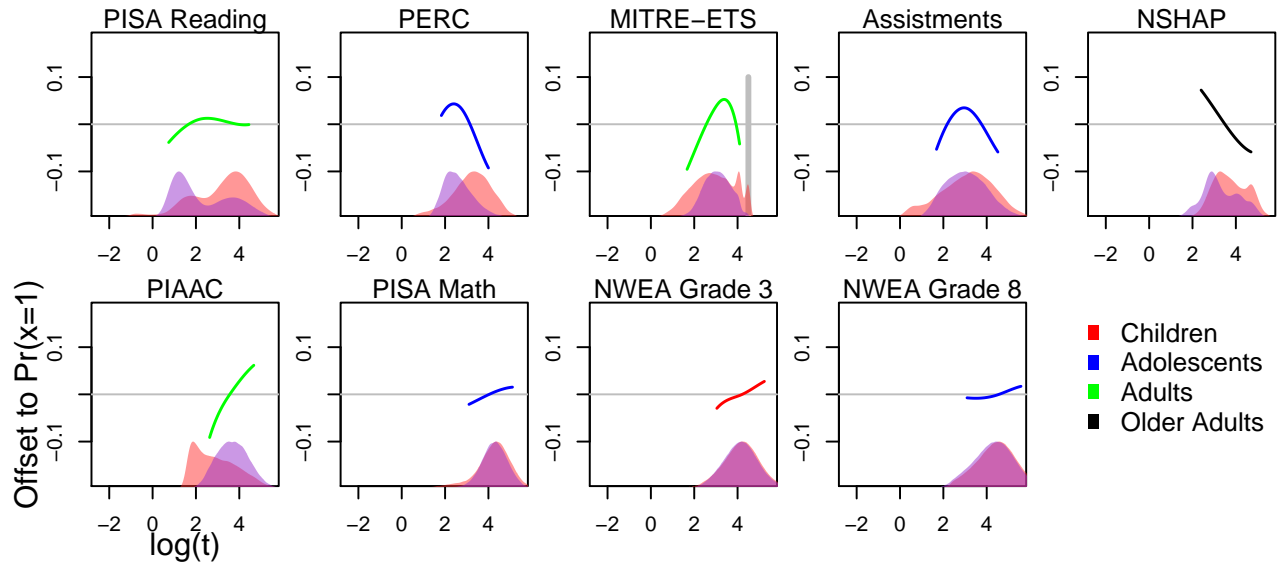

- Children

- Adolescents

- Adults

- Older Adults 
Figure 4: Estimated change in accuracy as a function of both response time (x-axis) and $p_{0}$ (y-axis). Colors can be interpreted based on legend on right. Blue indicates points where a marginal increase in time spent by a respondent on an item is expected to increase accuracy; red indicates points where the opposite is true. A lack of color represents a point with no estimated association between marginal increase in response time and accuracy.
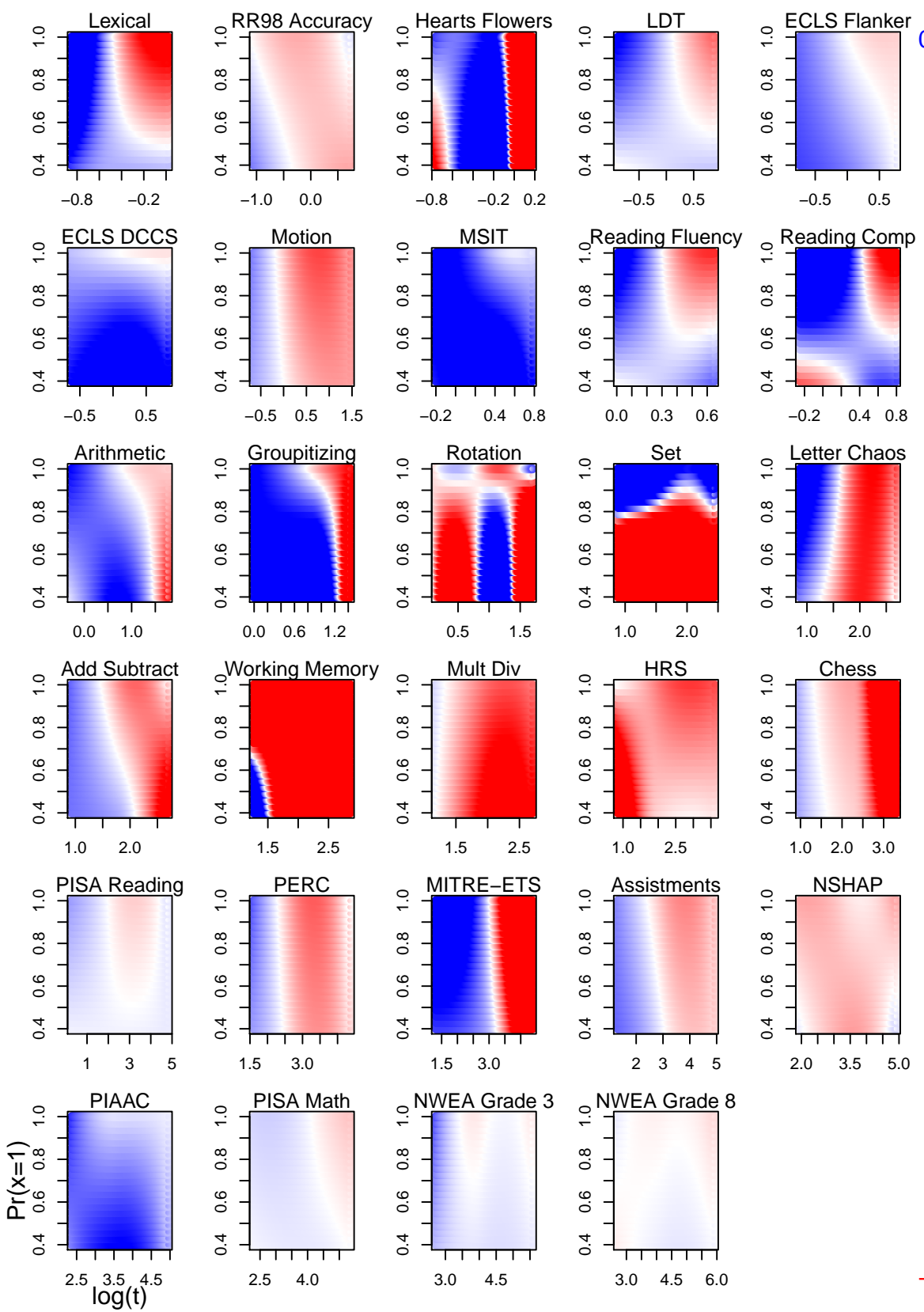

$0.25+$

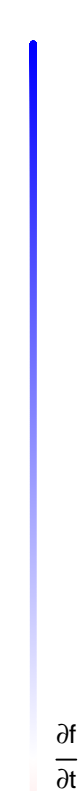

$\frac{\partial f}{\partial t}$

$\log (t)$

(3.0. 
Figure 5: Comparison of out-of-sample predictions (via $\exp (\bar{\ell})$; see Section 2.2.4) in 10\% hold-out. Predictions are made based on predictors shown in the legend. (A) is based on the overall mean accuracy in the data (see Figure 2). (B) is based on the mean accuracy for each item. (C) is based on the mean accuracy for each person. (D,E) are based on the mean standardized response time for each person (with E focusing just on correct responses). (F) combines $\mathrm{C}$ and $\mathrm{D}$.

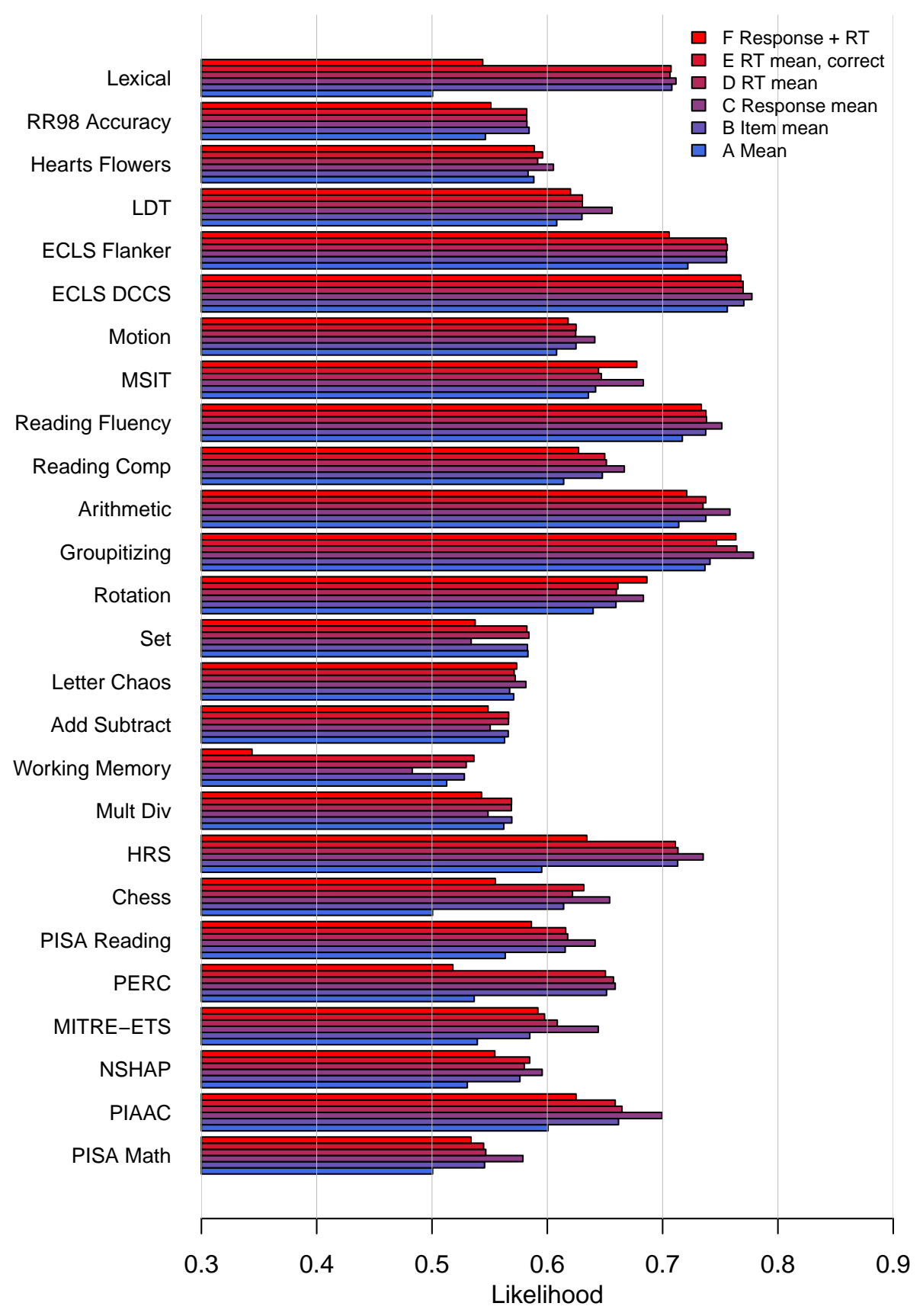

